Nervenkompressionssyndrome

\section{Schlafqualität beim Karpaltunnelsyndrom: Was bringen Schiene und Operation?}

Rubin $\mathrm{G}$ et al. The effectiveness of splinting and surgery on sleep disturbance in carpal tunnel syndrome. J Hand Surg Eur Vol 2018; 43: 286 289

Viele Patienten mit einem Karpaltunnelsyndrom leiden an nächtlichen Schlafstörungen. Inwiefern bessert sich durch das Tragen einer Schiene während der Nachtruhe bzw. nach einem operativen Eingriff die Schlafqualität? Dieser Frage sind israelische Wissenschaftler im Rahmen einer prospektiven Studie nachgegangen.

Teilnehmer waren 13 Frauen und 8 Männer im Alter zwischen 25 und 77 Jahren mit einem klinisch sowie elektrophysiologisch diagnostizierten, unilateralen Karpaltunnelsyndrom. In allen Fällen war ein chirurgischer Eingriff geplant. Die Ausschlusskriterien umfassten Patienten mit einer sekundären Karpaltunnel-Problematik, bspw. aufgrund eines Diabetes mellitus, einer rheumatischen Erkrankung, einer Hypothyreose oder eines vorangegangenen Handgelenktraumas. Auch Personen mit einer begleitenden zervikalen Radikulopathie, einer radioulnaren Neuropathie, einer vorangegangenen Operation eines Karpaltunnelsyndroms sowie Personen mit anderen Schlafstörungen gingen nicht in die Analyse ein. Das nächtliche Schlafverhalten der Probanden wurde zunächst ohne Therapie sowie während einer mehrtägigen Schienenversorgung beurteilt. Hierzu beantworteten die Studienteilnehmer den „Insomnia Severity Index“, hielten ihre subjektiven Eindrücke in einem Schlaftagebuch fest und trugen nachts am Handgelenk ein sogenanntes Aktometer, ein Messinstrument, das Bewegungsaktivitäten registriert und somit Rückschlüsse auf die Schlafqualität des Trägers erlaubt. Nach dem operativen Eingriff wurden dieselben Parameter erneut über mehrere Tage erhoben.
Ergebnisse Gemäß Insomnia Severity Index litten 18 der 21 Patienten initial an einer Beeinträchtigung des Nachtschlafs. Das größte Problem stellten Durchschlafstörungen sowie Beeinträchtigungen der Leistungsfähigkeit während des Tages dar. Einschlafstörungen oder ein frühes Erwachen empfanden dagegen nur wenige Studienteilnehmer als belastend. Mit Ausnahme eines Patienten berichteten alle Studienteilnehmer nach der Schienenversorgung über eine signifikante Verbesserung der Schlafqualität. Nach der Operation kam es bei allen Probanden zu einer weiteren signifikanten Verbesserung des Insomnia Severity Index-Scores. Diese Ergebnisse bestätigten sich auch bei der Auswertung der Schlaftagebücher: Während der Schienen-freien Studienphase stellten die Wissenschaftler eine signifikant schlechtere Schlafqualität sowie eine signifikant höhere Zahl von Schlafunterbrechungen fest als während der Phase mit Bandagenversorgung. Im Vergleich zur Schienenversorgung besserten sich diese $\mathrm{Pa}$ rameter nach der Operation jedoch nur unwesentlich. Bezüglich der AktigrafieBefunde zeigten sich keine signifikanten Unterschiede zwischen den 3 Behandlungsphasen.

\section{FAZIT}

Die Schienenversorgung beim Karpaltunnelsyndrom wirkt sich günstig auf den Nachtschlaf aus, schlussfolgern die Autoren. Im Hinblick auf den objektiven Insomnia Severity Index führt die operative Therapie zu einer weiteren Verbesserung der Schlafqualität. Einschränkend weisen sie auf die geringe Fallzahl der Studie hin und geben zu bedenken, dass ausschließlich Patienten mit einem ausgeprägten Karpaltunnelsyndrom, nicht jedoch Personen mit leichten Beschwerden an der Untersuchung teilnahmen.

Dr. med. Judith Lorenz, Künzell 